\title{
Transcriptome comparison of dengue-susceptible and -resistant field derived strains of Colombian Aedes aegypti using RNA-sequencing
}

\author{
Heather Coatsworth ${ }^{1,}$, Paola A Caicedo ${ }^{2}$, Geoffrey Winsor ${ }^{3}$, Fiona Brinkman ${ }^{3}$, \\ Clara B Ocampo ${ }^{2}$, Carl Lowenberger ${ }^{1 /+}$ \\ ${ }^{1}$ Simon Fraser University, Department of Biological Sciences, C2D2 Research Group, Burnaby, BC, Canada \\ ${ }^{2}$ Universidad Icesi, Natural Science Faculty, Centro Internacional de Entrenamiento e Investigaciones Médicas, \\ Department of Biology, Vector Biology and Control, Cali, Colombia \\ ${ }^{3}$ Simon Fraser University, Department of Molecular Biology and Biochemistry, Burnaby, BC, Canada
}

BACKGROUND Forty percent of the world's population live in areas where they are at risk from dengue fever, dengue hemorrhagic fever, and dengue shock syndrome. Dengue viruses are transmitted primarily by the mosquito Aedes aegypti. In Cali, Colombia, approximately $30 \%$ of field collected $A e$. aegypti are naturally refractory to all four dengue serotypes.

OBJECTIVES Use RNA-sequencing to identify those genes that determine refractoriness in feral mosquitoes to dengue. This information can be used in gene editing strategies to reduce dengue transmission.

METHODS We employed a full factorial design, analyzing differential gene expression across time (24, 36 and $48 \mathrm{~h}$ post bloodmeal), feeding treatment (blood or blood + dengue-2) and strain (susceptible or refractory). Sequences were aligned to the reference $A$ e. aegypti genome for identification, assembled to visualize transcript structure, and analyzed for dynamic gene expression changes. A variety of clustering techniques was used to identify the differentially expressed genes.

FINDINGS We identified a subset of genes that likely assist dengue entry and replication in susceptible mosquitoes and contribute to vector competence.

MAIN CONCLUSIONS The differential expression of specific genes by refractory and susceptible mosquitoes could determine the phenotype, and may be used to in gene editing strategies to reduce dengue transmission.

Key words: dengue - Aedes aegypti - yellow fever mosquito - RNA sequencing - refractory mechanisms - innate immunity

Vector-borne pathogens are responsible for a significant proportion of the world's most debilitating and devastating human diseases. ${ }^{(1)}$ In their role as vectors of protozoans, viruses, and nematodes, mosquitoes are the indirect cause of more than 2 million deaths annually. ${ }^{(2)}$ Of these, dengue is the most widespread arthropod-borne virus (arbovirus) disease, infecting up to 390 million people each year throughout tropical and subtropical regions. ${ }^{(1,2)}$ Dengue is transmitted primarily by Aedes aegypti, and to a lesser extent by Aedes albopictus. Changes in global travel, urbanization, and climate have facilitated the expansion of Ae. aegypti populations and consequently has allowed dengue to thrive. Half of the world's population is at risk of contracting dengue, a statistic that could increase as the effects of climate change mount. ${ }^{(2)}$

doi: 10.1590/0074-02760200547

Financial support: COLCIENCIAS (Contract 2229-519-28645 to CB), an NSERC Discovery grant to CL (RGPIN261940), Emerging Leaders in the Americas Program (ELAP) scholarships to PC, and Graduate Fellowships (SFU) and the HR MacCarthy Scholarship (SFU) to HC.

$\mathrm{HC}, \mathrm{PAC}$ and $\mathrm{CL}$ contributed equally to this work.

*Current address: University of Florida, Department of Infectious Diseases and Immunology, Gainesville, FL.

+ Corresponding author: clowenbe@sfu.ca

(1) https://orcid.org/0000-0002-1839-359X

Received 17 October 2020

Accepted 28 April 2021
Although insecticides, larvicides, and source reduction are used widely to reduce mosquito populations, none seem able to dampen dengue transmission significantly. This has resulted in an emphasis on mosquito bio-manipulation or genetic modification techniques to induce sterility, decrease lifespan, or reduce vector competence. (3) These applications are based on understanding the molecular interactions between vector and virus, as well as basal vector genetics, and have shown great promise in developing new and effective vector control strategies.

Although Ae. aegypti is the principal vector of dengue viruses (DENVs), not all Ae. aegypti females transmit the virus. In Cali, Colombia, approximately $30 \%$ of field collected Ae. aegypti are refractory to all four dengue serotypes ${ }^{(4,5,6,7,8)}$ through one or more of the established barriers to flavivirus development; a midgut infection barrier (MIB) in which DENV is unable to replicate within midgut cells, or a midgut escape barrier (MEB) in which the virus cannot escape the midgut cells. Other barriers include a salivary gland infection barrier (SIB) in which the virus cannot enter the salivary glands, or a salivary gland escape barrier (SEB) in which DENV is unable to disseminate into the salivary gland lumen. ${ }^{(9)}$ These refractory mechanisms and overall innate immune responses to DENV have been studied principally in long established, specifically selected laboratory strains of Ae. aegypti. ${ }^{(10,11)}$ In Cali, Colombia we can collect mosquitoes in the field with one of three phenotypes; susceptible (Cali-S), refractory with a midgut infection barrier (Cali-MIB) and refractory with a 
midgut escape barrier (Cali-MEB). All three phenotypes can be collected within the same communities, and inside the same houses or oviposition sites within different neighborhoods. These have been raised in the laboratory and selected to increase the proportion of each phenotype, giving rise to the field derived strains. ${ }^{(8)}$

We have described significant differences in the expression of apoptosis related genes in the Cali-MIB and Cali-S strains. ${ }^{(6,7,8)}$ Knocking down apoptosis related genes altered the phenotype of Ae. aegypti, but could only explain $\sim 30 \%$ of the refractory phenotype. ${ }^{(7,8)}$ We carried out a midgut transcriptome analysis, using RNA sequencing (RNA-seq) technology, to identify all transcripts in the midguts of Cali-S and CaliMIB females at three different time points (24, 36 and $48 \mathrm{~h}$ post feeding) that are relevant to the period when the virus is entering, replicating in, and then exiting the midgut epithelial cells respectively. The aim of this study was to identify, in an unbiased manner, all differentially expressed genes that might contribute to the refractory or susceptible phenotypes.

Other studies have examined gene expression profiles after exposure to DENV in laboratory colonies of Ae. aegypti that are susceptible to $\mathrm{DENV},{ }^{(9,10,11)}$ and some have investigated semi-refractory laboratory strains. $(9,10,12,13,14)$ Our study is unique in that it analyzes mosquitoes that have evolved the refractory and susceptible phenotypes in the field with no human directed laboratory selection specifically for refractoriness or susceptibility to DENV.

\section{MATERIALS AND METHODS}

Ethics statement - All female mosquitoes were exposed to dengue virus through an artificial membrane feeder. Adults in colonies were fed on hamsters at CIDEIM (Cali, Colombia) under protocols approved by the CIDEIM institutional review committee for research in animals (CIEIA).

Mosquito rearing - The collection, rearing and selection of the Cali-S and Cali-MIB strains of Ae. aegypti have been described. ${ }^{(8)}$ These strains were maintained under standard laboratory conditions: $28 \pm 2^{\circ} \mathrm{C}, 70 \%$ relative humidity, and a 12:12 h light-dark cycle. Adults were supplied with a $10 \%$ sugar solution ad libitum.

Virus propagation and mosquito infections - DENV2 (New Guinea C strain) was propagated in Ae. albopictus (Skuse) C6/36HT cells. Infected cells were incubated for 14 days at $32^{\circ} \mathrm{C}$ in $\mathrm{L} 15$ medium supplemented with $2 \%$ heat-inactivated fetal bovine serum (FBS), $1 \%$ peni- cillin/streptomycin, and 1\% L-glutamine. Virus and cells were harvested and collected in a $15 \mathrm{~mL}$ conical centrifuge tube. The viral suspension was mixed 1:1 with defibrinated rabbit blood to create an infectious blood meal. Aliquots of the infected cell suspension, and the mixture of blood and virus were titred before and after the infection process as described previously. ${ }^{(8)}$ Titers in the cell suspensions ranged from $10^{8}$ to $10^{8.5} \mathrm{TCID}_{50} / \mathrm{mL}$ in all oral challenges. Five to eight-day old adult female Cali$\mathrm{S}$ and Cali-MIB mosquitoes were exposed for $2 \mathrm{~h}$ to the infectious blood meal via an artificial membrane feeder. (7) All infections were carried out in Bio Safety Level 2+ facilities. After exposure to a blood meal with or without DENV-2, females that had fed to repletion were transferred to $300 \mathrm{~mL}$ containers, covered with mesh $(\sim 20$ mosquitoes/container), and were given access to $10 \%$ sucrose solution ad libitum. Containers were maintained under the laboratory conditions described above.

Mosquito dissections - Midguts from $\mathrm{F}_{15}$ adult females were dissected from each strain (Cali-S and CaliMIB) under each feeding treatment (blood meal or blood meal with DENV) and at each time point [24, 36 and $48 \mathrm{~h}$ post blood meal (PBM)] (Table I). Any remaining blood in the midgut was removed during dissection and the tissues were rinsed in 1X phosphate-buffered saline (PBS). In order to obtain enough RNA, midguts from three biological replicates were pooled for each of the 12 treatment groups. All dissections were performed in diethylpyrocarbonate (DEPC) sterile water on a cold table, and dissected tissues were immediately transferred to a microcentrifuge tube containing $200 \mu \mathrm{L}$ of RNAlater ${ }^{\circledR}$ Stabilization Solution (Ambion, Austin, Texas). All samples were subsequently transported from CIDEIM (Cali, Colombia) to Simon Fraser University (Burnaby, British Columbia), and stored at $-20^{\circ} \mathrm{C}$.

$R N A$ extraction, library preparation, and RNA sequencing - Total RNA was extracted from each pool of midguts and carcasses using Trizol (Sigma, Oakville, Ontario) as per the manufacture's protocols. RNA concentrations were determined using a spectrophotometer (NanoDrop, ND-1000). Poly-A mRNA purification was performed with the Micro Poly A Purist Kit (Ambion, Austin, Texas) following the manufacturer's protocols. From each mRNA sample, $100 \mathrm{ng}$ was used to generate cDNAs using the Ultra RNA Library Prep Kit for Illumina (New England BioLabs, Ipswich, Massachusetts). All purification reactions were completed using AMPure XP Beads (Beckman Coulter, Brea, California). Fragment length analyses and overall library quality were complet-

TABLE I

Full factorial treatment design outlining all twelve experimental treatments $(n=36$ for each treatment)

\begin{tabular}{|c|c|c|c|c|c|c|c|c|c|c|c|c|}
\hline \multirow{3}{*}{$\begin{array}{l}\text { Hours PBM } \\
\text { Treatment } \\
\text { Strain Cali- }\end{array}$} & \multicolumn{4}{|c|}{24} & \multicolumn{4}{|c|}{36} & \multicolumn{4}{|c|}{48} \\
\hline & \multicolumn{2}{|c|}{ Blood } & \multicolumn{2}{|c|}{ Blood + DENV-2 } & \multicolumn{2}{|c|}{ Blood } & \multicolumn{2}{|c|}{ Blood + DENV-2 } & \multicolumn{2}{|c|}{ Blood } & \multicolumn{2}{|c|}{ Blood + DENV-2 } \\
\hline & $\mathrm{S}$ & MIB & $\mathrm{S}$ & MIB & $\mathrm{S}$ & MIB & $\mathrm{S}$ & MIB & $\mathrm{S}$ & MIB & $\mathrm{S}$ & MIB \\
\hline
\end{tabular}

DENV: dengue viruses; MIB: midgut infection barrier; PBM: post blood meal. 
ed on the final libraries at $2 \mathrm{nM}$ using a Bioanalyzer (Agilent High Sensitivity Chip, Agilent Santa Clara, California). Libraries were sequenced at 100X depth as technical duplicates across multiple lanes using an Illumina miSeq platform at Fusion Genomics (Burnaby, BC).

Processing of raw sequencing reads - A basic bioinformatics workflow was modified to accommodate newer programs and multiple analyses (Fig. 1). A complete list of all bioinformatic resources can be found in Supplementary data (Table I). The quality of the sequence data from each of the 12 treatments was checked using FastQC (v. 0.11.1), and a sequence trimmer, Trimmomatic (v. 0.30), was used on each of the 12 files to reduce overrepresented sequences, as well as to remove sequences less than $90 \mathrm{bp}$ in length.

Read alignment and mapping - The Ae. aegypti genome and associated gene annotation files were obtained from VectorBase (http://www.vectorbase.org): AaegL3 Scaffolds was used as the genome, while AaegL3 Basefeatures was used for gene annotation. Tophat2 (https:// tinyurl.com/yyxv8r3a) was used to align and map reads. Samstat (v.1.09) (https://tinyurl.com/yy9b7t5z) subsequently was used to check mapping quality.

Differential expression tests - Twenty-four separate differential expression (DE) tests were run to investigate the effects of time, viral presence and mosquito strain [Supplementary data (Table II)]. Because our MIB strain is only $\sim 50 \%$ refractory, we assumed, based on Caicedo et al., ${ }^{(8)}$ that $50 \%$ of the refractory mosquitoes were indeed phenotypically refractory. As such, strain comparisons were made between a pool of susceptible mosquitoes, and a pool of half refractory and half susceptible mosquitoes. Three different programs were used to analyze the RNA-seq data. Cuffdiff (v. 2.2.1) was used to test differential expression at both the gene and transcript level. Cuffdiff differential expression tests without replicates were run using the 'blind' method, while tests with replicates were run using the 'pooled' method. DESeq2 (v. 1.16.0), was also used to test for differential expression at the gene level. Tests without biological replicates were run under the 'blind' method, 'fit-only' sharing mode, and the 'parametric' fit-type, while tests with replicates were run using the 'pooled' method, the 'maximum' sharing mode, and the 'parametric' fit-type.

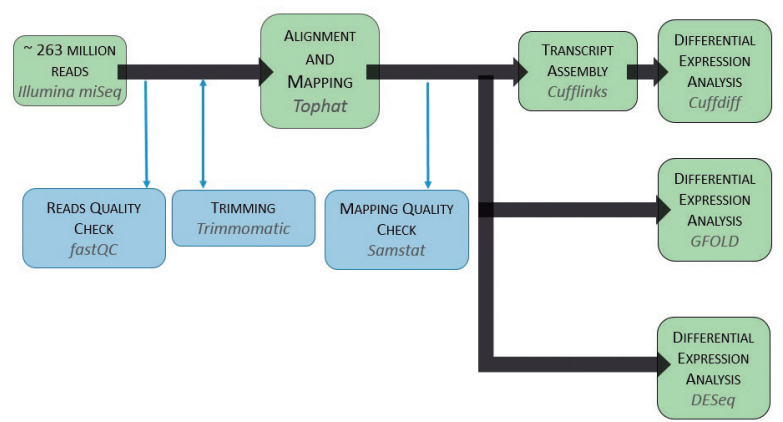

Fig. 1: modified and adapted RNA rocket Galaxy portal bioinformatics workflow.
Both programs generate a $p$-value from analyzing if the variance present in a group of samples is beyond what is expected from a simple Poisson model of the RNA sequencing data. Fold change values from Cuffdiff and DESeq2 are generated from Fragments Per Kilobase of transcript per Million mapped reads (FPKM) values, specifically, $\log _{2}\left(\mathrm{FPKM}_{\text {samplel }} / \mathrm{FPKM}_{\text {sample }}\right)$. A third differential expression program, GFOLD (v. 1.1.1), which is specifically designed for RNA-seq analyses without replicates, was used with default parameters. GFOLD reports a GFOLD value, which acts as a reliable $\log _{2}$ fold change value, calculated using Reads per Kilobase of transcript per Million mapped reads (RPKM) values. GFOLD values of zero show no differential expression. Sequences of differentially expressed genes were linked with annotations corresponding to gene names, GO (Gene Ontology) terms, and KEGG (Kyoto Encyclopedia of Genes and Genomes) terms in order to facilitate downstream over-representation analysis (annotations obtained using the Biomart tool at VectorBase).

Analysis of DE data - Two separate sub-analyses were completed on each DE output; one included only differentially expressed genes, and another that contained immune-related genes that were not differentially expressed statistically, but which the literature has indicted that even small changes in gene expression can have serious biological implications. To complete the immune specific analysis, an Ae. aegypti specific immune related list of genes was downloaded from ImmunoDB [Supplementary data (Table III)]. Functional classifications were assessed using a concatenated list of GO terms obtained through ImmunoDB. The second analysis was performed in a similar manner, excluding the ImmunoDB gene filtering step.

Clustering analyses and functional enrichment tests were completed on data obtained from all treatments, without incorporating a variance scaling factor, as data were found to be homoscedastic [Supplementary data (Fig. 1)]. To investigate how closely the expression profiles from each sample compared, a principal component analysis (PCA) plot using Euclidian distances was created using DESeq 2 by log transforming the merged read count. Ae. aegypti GO terms were used to complete functional over-representation analyses via Ontologizer (v. 2.0). Two main types of clustering were performed through $\mathrm{R}(\mathrm{v}$. 3.1.1): hierarchical and partitioning (k-means) clustering. Dendrogram cutting was used to determine the optimal number of clusters for k-means clustering.

Validation of differential gene expression using quantitative polymerase chain reaction (PCR) - Droplet digital PCR (ddPCR) and quantitative real time PCR (qPCR) were used to validate expression values from RNA-seq. Validation tests were completed on cDNAs generated from three independently generated biological replicates from different generations of mosquitoes than those used to create the RNA-seq libraries. Thermocycling conditions for ddPCR were: $95^{\circ} \mathrm{C}$ for $10 \mathrm{~s}, 55^{\circ} \mathrm{C}$ for $10 \mathrm{~s}$, and $72^{\circ} \mathrm{C}$ for $30 \mathrm{~s}$ in $20 \mu \mathrm{L}$ reactions (containing $1 \mu \mathrm{L}$ of cDNA) using QX200 ddPCR EvaGreen Supermix (BioRad Laboratories, Hercules, California, USA) on an au- 
tomated QX200 Droplet Digital PCR System (Bio-Rad Laboratories, Hercules, California, USA). QuantaSoft v1.7.4 (Bio-Rad Laboratories, Hercules, California, USA) was used to obtain an absolute expression quantification. qPCR was performed on a Light Cycler $^{\circledR} 96$ system (Roche, Basel, Switzerland) using PerfeCTa SYBR ${ }^{\circledR}$ (Quantabio, Massachusetts, USA). Thermocycling conditions for qPCR were: $95^{\circ} \mathrm{C}$ for $10 \mathrm{~s}, 55^{\circ} \mathrm{C}$ for $10 \mathrm{~s}$, and $72^{\circ} \mathrm{C}$ for $30 \mathrm{~s}$ in $10 \mu \mathrm{L}$ reactions (containing $4 \mu \mathrm{L}$ of $1: 50$ diluted cDNA). LightCycler ${ }^{\circledR} 96$ Application Software Version 1.1.1 (Roche, Basel, Switzerland) was used to obtain relative gene expression comparisons against a constitutively expressed housekeeping gene, $40 \mathrm{~S}$ ribosomal protein RPS17 (AAEL004175). Comparisons between ddPCR and $\log _{2}$ qPCR values and RNA-seq GFOLD001 values were made, noting the direction and magnitude of change. All RNA-seq GFOLD001 values were divided by the corresponding ddPCR or qPCR values in order to test the similarity between the two datasets.

\section{RESULTS}

Raw sequencing reads processing, alignment and mapping - Each RNA-seq library generated between 14 and 28 million reads $>90 \mathrm{bp}$ for each of the 12 treatments (Table I). Eighty three percent of all reads mapped to the genome (17\% unmapped), and $63 \%$ of the mapped reads had an error rate of less than $0.001 \%$ [Supplementary data (Table IV).

Differential expression analysis - All three programs identified the same differentially expressed genes. All time point comparisons under the Cali-S virus fed versus Cali-MIB virus fed test yielded similar functional group profiles (Fig. 2). Diverse and unknown functional groups represented the largest proportion of genes, followed by transcription/translation, transport, metabolism, redox/ stress/mitochondrial and finally the immune group. The remaining groups each represented less than three percent of the total number of differentially expressed genes.

Statistical and systems analysis of differential expression data - Hierarchical clustering produced a dendrogram (Fig. 3), with a clear separation between treatments analyzed at the 48-h time point (right branch) and all other treatments (left branch). The further splits within this right branch, were based on the viral treatment of the sample (either blood fed or blood and virus fed). The left branch however displayed an initial splitting of the Cali-S and Cali-MIB strains, with further branching into separate blood fed and virus fed treatments, and a final branching event into the 24 and $36 \mathrm{~h}$ time points.

The functional over-representation analysis again highlighted the $48 \mathrm{~h}$ time point as more diverse and dissimilar to the other time points. There was a large number of terms associated with cellular localization and transport across all comparisons. Comparisons at earlier time points ( 24 and $36 \mathrm{~h}$ ) represented a generation of precursor metabolites, envelope proteins, and ion binding; while later time points ( 36 and $48 \mathrm{~h}$ ), invoked intracellular signal transduction and small molecule metabolic processes [Supplementary data (Table V)].
Based on dendrogram cutting, the k-means analysis [Supplementary data (Fig. 2)] clustered all the gene count data into seven distinct clusters. The first cluster represented $98 \%$ of the genes $(17,254)$, and as such, was tied to a wide variety of functional classes. Cluster 2 (44 genes) was primarily associated with ribosomal intracellular and translation functions, as well as RNA transport and degradation activity. Cluster 3 (one gene, AAEL013284) was specifically related to serine-type peptidase activity, as was cluster 7 (one gene, AAEL007818), and cluster 6 (10 genes). Similar to cluster 2, cluster 4 (162 genes) contained genes with many ribosomal functions, as well as functions associated with ATP binding and transport, metabolic pathways, and carbohydrate metabolism. Finally, cluster 5 (seven genes) was solely made up of genes representing metallopeptidases, GTP binding and GTPase activity.

Choosing a candidate gene shortlist - From these data, we further selected 15 differentially expressed genes (Table II) for further study based on three criteria: (i) highly differentially expressed, (ii) highly differential-

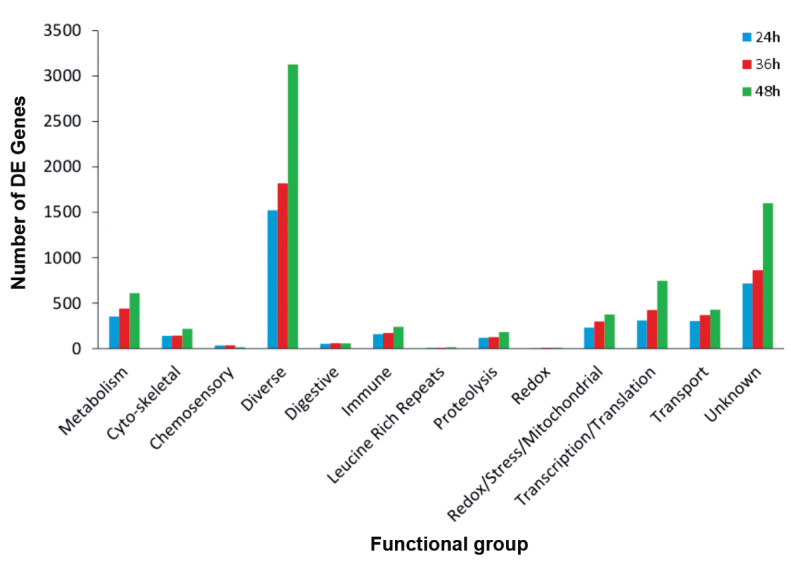

Fig. 2: significantly differentially expressed genes between Cali-S and Cali-MIB strains at 24,36 and $48 \mathrm{~h}$ after ingesting dengue virus serotype 2 , arranged by broad functional groups denoted by ImmunoDB.

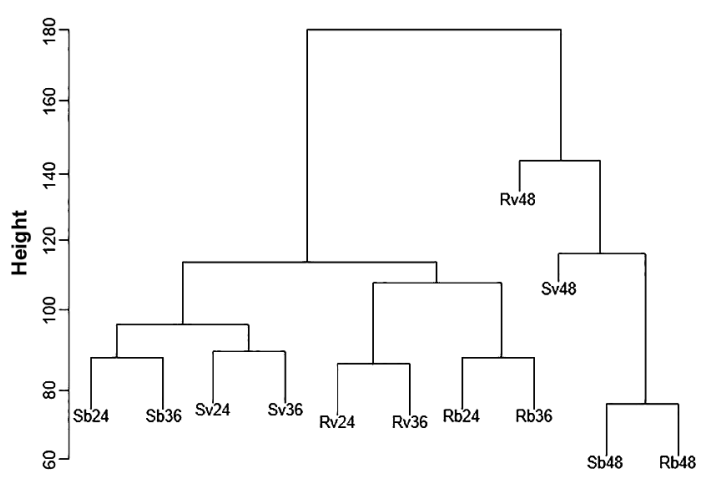

Fig. 3: consensus hierarchical clustering result from DESeq2 (v. 1.16.0), Cuffdiff (v. 2.2.1) and GFOLD (v. 1.1.1) generated using R (v. 3.1.1). Clustering shows the phylogenetic relationship between all 12 treatment expression profiles (S: Cali-S, R: Cali-MIB, v: virus fed, b: blood fed, numbers represent time points). Euclidian distances were generated to compute Complete Linkage clustering. 
ly expressed and immune related, and (iii) immune-related genes tagged in other published research papers (Table III). Genes were chosen from both the top significantly DE list, as well as the DE immune list. Only genes with documented or putative unique functions were chosen. Genes were classified as up-regulated when expression was higher in virus fed Cali-MIB versus virus fed Cali-S or blood fed Cali-MIB. Conversely, genes were labeled as down-regulated when expression was higher in virus fed Cali-S versus virus fed Cali-MIB or blood fed Cali$\mathrm{S}$. Up-regulated genes might be expressed to block viral cell entry and exit, stop replication or aid in immune viral clearance, while down-regulated genes may do the opposite, aiding in DENV entry, exit, and replication.

Differential expression validation - Four candidate genes (autophagy related target of rapamycin, TOR, AAEL000693, a 40S ribosomal gene, AAEL013694, a low-density lipoprotein receptor gene, AAEL014222, and a bumetanide-sensitive $\mathrm{Na}-\mathrm{K}-\mathrm{Cl}$ co-transport, AAEL009888), and five non-candidate genes (60 S ribosomal protein L15, AAEL012736, 60 S ribosomal protein L35a, AAEL000823, Eukaryotic translation initiation factor 3 subunit G, AAEL012661, an uncharacterized gene, AAEL002930, and 4-nitro, AAEL007097) were chosen as representatives for differential expression validation. ddPCR was used to validate candidate genes, as their expression levels were lower and this technique is more sensitive than qPCR. Non-candidate gene validation was completed using qPCR [Supplementary data (Fig. 3)], as the overall expression levels of these genes was high enough for reliable detection. Any genes that differed in direction of level of differential expression between RNAseq and ddPCR analyses (AAEL013694, AAEL014222 and AAEL000693) were removed from the candidate gene list.

\section{DISCUSSION}

Differential expression analysis - All three methods (Cuffdiff, DESeq2 and GFOLD) identified the same top differentially expressed genes. Cuffdiff and DESeq2 are both extremely conservative in their list of differentially expressed genes compared to GFOLD. It is not uncommon for immunologically relevant genes to rank below others in the list of the greatest differentials in gene expression. This trend is further highlighted in immune genes, where even slight changes in expression may have large downstream effects. As such, the ordering of gene expression differences (from highest to lowest) may have resulted in ranking biologically relevant genes lower in importance based solely on expression level differences. To overcome this quandary, two lists of candidate genes were generated: one based on the top DE genes (shared amongst all three programs), and one based on the most expressed immune related genes (as identified by ImmunoDB, still shared amongst all three programs).

There were some general trends within the dataset. Both Cali-S and Cali-MIB mosquitoes infected with DENV-2 had increased expression of digestive genes such as trypsins, serine endopeptidases and metalloproteinases compared with their counterparts fed solely on blood. These digestive enzymes are likely important early regulators of infection; ${ }^{(15)}$ an increase in these digestive enzymes could assist in dampening the ability of DENV to enter and replicate in cells, as the level of viral degradation within the midgut could be higher.

In Cali-MIB females exposed to DENV, we observed higher levels of metalloproteinases (MMPs), as well as increases in the expression of a Niemann-pick type $\mathrm{C} 2$ gene. Niemann-pick-C2 is a cholesterol transporter, and has been identified in various studies as a viral agonist that may enhance, or be required for, the entry of DENV-2 into cells. ${ }^{(16)}$ However, our results in refractory mosquitoes seem to suggest the opposite. It is possible, that in response to other mechanisms expressed to decrease viral titres in the midgut of refractory mosquitoes, that DENV upregulates Niemann-pick $\mathrm{C} 2$ expression to remain viable.

In Cali-S females, we see higher expression levels of several odorant binding proteins (OBPs) (AAEL006176OBP27, AAEL002606-OBP35, AAEL012377OBP55, AAEL009449-OBP39, AAEL010666-OBP42, AAEL013018-OBP56) as well as an anti-apoptosis gene (AAEL009074-Inhibitor of Apoptosis, AeIAP1). The role of OBPs in the midgut is unclear, although it has been proposed that they act as signalling mechanisms for odorant binding proteins in the salivary glands, ${ }^{(17)}$ inducing the mosquito to bite repeatedly and enhancing virus transmission. AeIAPl, on the other hand, is involved in inhibiting apoptosis, and has been characterized as being pro-viral, preventing infected cells from undergoing apoptosis and eliminating the virus before it replicates. Unfortunately, knock-downs of AeIAP1 were lethal to the mosquitoes, and cannot be the sole mechanism driving refractoriness in the Cali-MIB strain.

There were notable temporal differences in the expression of genes within each treatment and strain. Most digestive function ontology terms correlate directly with mosquito blood meal processing. We observed trypsins and sodium and potassium co-transporters at $24 \mathrm{~h}$ post blood meal, serine endopeptidases, carboxypeptidases and lipases at $36 \mathrm{~h} \mathrm{PBM}$, and heme peroxidases, cytochrome $\mathrm{p} 450 \mathrm{~s}$ and sucrose transporters at $48 \mathrm{~h}$ PBM. Insects rapidly produce digestive enzymes upon feeding, and these decrease in production as absorption occurs within the midgut. ${ }^{(18)}$ Specifically, late trypsin is activated $12-48 \mathrm{~h}$ post blood meal, during which lipid digestion occurs via phospholipases and phosphatases, which hydrolyze ester bonds, solubilizing cell membranes for the passage of lipids into the hemolymph. ${ }^{(18)}$ This may result in the spike of expression in these enzymes at earlier time points, and lower expressions at later time points. The digestion of the blood meal produces toxic heme as a by-product, and this toxic heme has specific binding sites on the peritrophic matrix where it is bound and excreted after blood digestion has occurred. As a result, heme cannot interact with and damage the midgut epithelial cells. Mosquitoes also use p450-like enzymes such as CYP6 and CYP9 to assist in heme detoxification. ${ }^{(18)}$ This is likely why higher levels of heme peroxidases and cytochrome p450s start to appear around $48 \mathrm{~h}$ PBM. Sucrose transporters may have higher expression levels at $48 \mathrm{~h}$ as the mosquito is likely in the process of digesting, and subsequently transporting, these carbohydrates. 


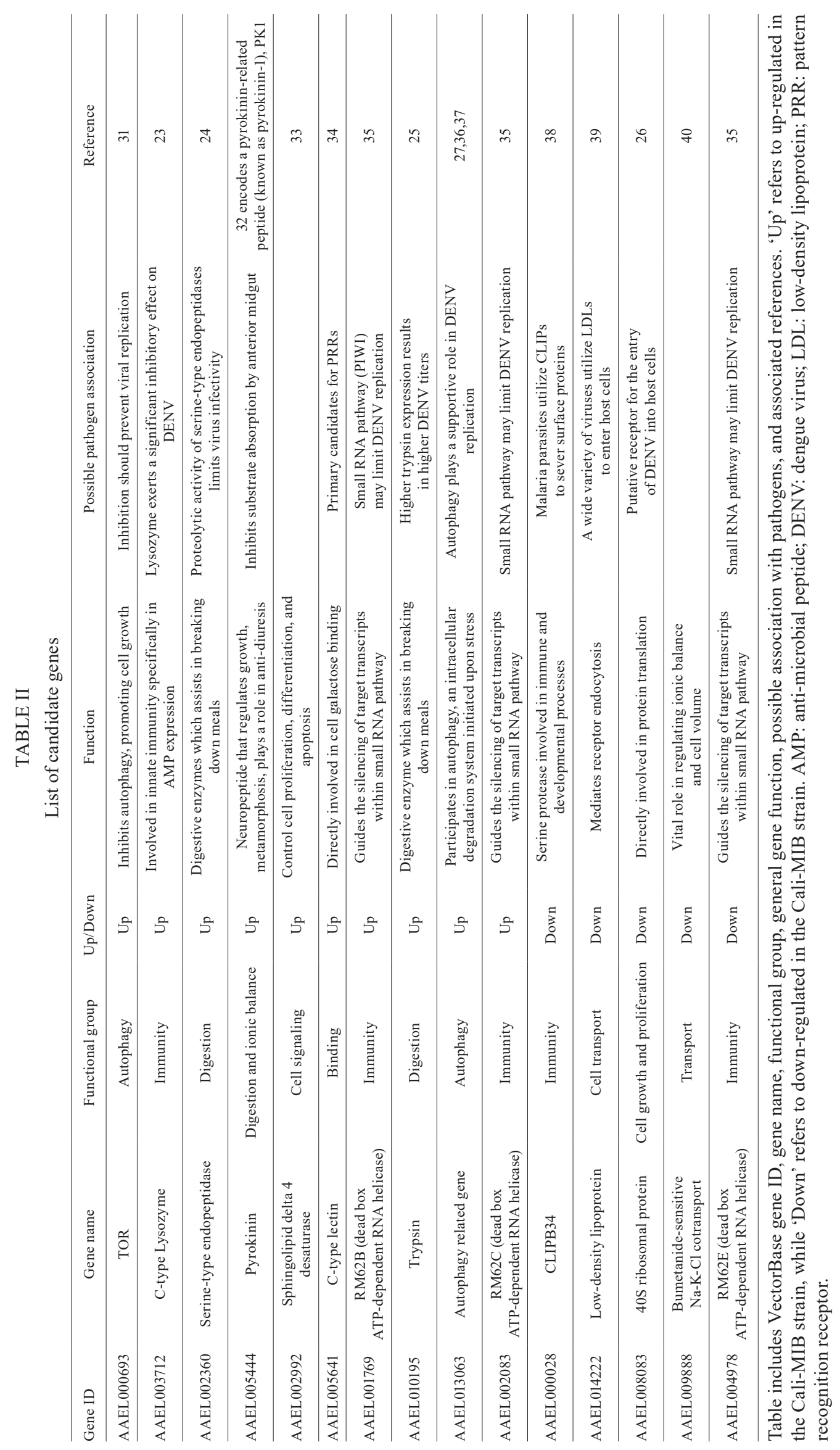


Eighty five of the genes identified in this RNA sequencing study have been implicated in previous refractory mosquito expression studies, ${ }^{(10,12,13,19)}$ including a microarray study on Cali-MIB and Cali-S at one time point only; $30 \mathrm{~h}$ after blood feeding. ${ }^{(20)}$ These correlations centered on digestive genes such as trypsins and serine-endopeptidases, as well as signalling and cell entry genes such as lectins and lipoproteins. A smaller subset of immune related genes was also common, including a variety of anti-microbial peptides, CLIP (Nterminal to the chymotrypsin serine protease domain, named due to its likeness in shape to a paperclip) domains, apoptotic genes, and small RNA pathway molecules. Although many genes were common between these datasets, some genes had differences in their direction of differential expression. These differences were mostly evident between our refractory strain (Cali-MIB) and the MOYO-D strain, and primarily encompassed cell signalling, processing and transport genes such as ubiquitin, dynein, adenylyl cyclase, clathrin, dishevelled, and multiple vitellogenin precursors.

A large proportion of differentially expressed genes are MMPs, which have a strong association with immunity. MMPs play important roles in pathogen infection, acting as both agonists and antagonists. In humans, increased MMP activity is associated with increased pathogenicity, as MMPs assist in breaking down the basal lamina of tissues, allowing for subsequent viral entry and replication, often resulting in increased vascular leakage. ${ }^{(21)}$ In mosquitoes, MMPS have been implicated in extracellular matrix remodelling, potentially allowing virions to pass through an altered basal lamina. (22) MMPs also have been annotated as anti-viral, acting as apoptotic effectors in the important anti-viral JAKSTAT pathway. ${ }^{(15)}$ In theory, decreasing these pervasive midgut specific MMPs would help insects create a tissue specific barrier to migrating pathogens. A down regulation of MMPs in Ae. aegypti was correlated with decreased viral titre, or elimination of DENV.$^{(9,13,19)}$

We have reported trends observed within the dataset. Some genes in the Ae. aegypti genome have not been annotated, and their expression will appear in the dataset as 'conserved hypothetical proteins' or 'hypothetical proteins', and as such, the role of these genes was not examined.

Statistical and systems analysis of differential expression data - The outputs from the hierarchical clustering and PCA analysis were, as expected, similar, as both involve distance matrix measures to scale and visualize data sets. In both analyses the 48 -h time point appears much more isolated than the 24- and 36-h time points, likely because the mosquito has finished or is near the end point of blood digestion, and thus different regulatory genes are at play. In Cali-S mosquitoes, by 48 $\mathrm{h}$, the virus will have migrated into and replicated within other mosquito tissues, and as such, different genes may be expressed. Conversely, DENV does not enter the hemocoel of Cali-MIB mosquitoes and therefore it is likely that these expression differences may be related to the process of viral elimination. Furthermore, the lack of significant differences in Cali-S and Cali-MIB females fed solely on blood at $48 \mathrm{~h}$ PBM suggests that the major differences between these strains are directly related to their response towards DENV.

The partitioning (k-means) analysis allowed us to view the functional clustering of the differentially expressed genes, while the Ontologizer completed a functional over-representation analysis. Both outputs yielded similar trends. We observed many genes with a wide variety of functions, with many genes at $24 \mathrm{~h}$ and 36 $\mathrm{h}$ PBM associated with blood meal processing, suggesting differences in blood meal digestion between the two mosquito strains. Many ribosomal, RNA transport and degradation terms clustered together, which could suggest dengue is utilizing host mechanisms to help in its own replication. Since the virus itself utilizes the host endoplasmic reticulum for transport and assembly, these changes in expression could reflect efficient viral infection and propagation.

Candidate gene analysis - Most of these candidate genes $(5 / 15)$ were immune related; a c-type lysozyme (AAEL003712), and three dead box ATP dependant RNA helicases (AAEL001769, AAEL002083, and AAEL 004978) may limit DENV replication as part of the mosquito's innate immune response, ${ }^{(23)}$ while CLIBB34 (AAEL000028) may be manipulated by the virus to interfere with cell surface proteins. Multiple digestive genes (a serine-type endopeptidase, AAEL002360, a pyrokinin, AAEL005444, and a trypsin, AAEL010195), also in the list, have been proposed to limit viral infectivity due to their high proteolytic activity and role in absorption, ${ }^{(24)}$ although some studies have shown the opposite to be true. ${ }^{(25)}$ Candidate genes related to cell signaling, growth, binding and transport (a sphingolipid delta 4 desaturase, AAEL002992, a $40 \mathrm{~S}$ ribosomal protein, AAEL008083, a c-type lectin, AAEL005641, and a low-density lipoprotein, AAEL014222, respectively) may play roles in assisting or inhibiting viral cell entry. (26) Lastly, two autophagy candidate genes, an autophagy related target of rapamycin, AAEL000693, and an autophagy related gene, AAEL013063, both up-regulated in Cali-MIB mosquitoes may play a role in viral replication. ${ }^{(27)}$ A summary of all our candidate genes, their function as well as possible DENV association can be found in Table II. We have focused on four of these genes (validated using ddPCR) in more detail below.

Although NaK (a bumetanide-sensitive Na-K-Cl cotransport, AAEL009888) has not been reported previously as important in the mosquito-dengue literature, it plays a vital role in regulating ionic balance and cell volume. NaK may be localized in the apical membrane of midgut epithelial cells in Ae. aegypti, as was demonstrated by an ortholog of AAEL009888 in Manduca sexta. ${ }^{(28)}$ Furthermore, sodium transporters are needed to maintain intracellular $\mathrm{pH}$, and changes in the expression of these transporters could result in changes to cell homeostasis. NaK could be necessary for the maintenance of intracellular homeostasis, and this could be why we see a higher expression of the NaK transcript in susceptible infected mosquitoes. 
TABLE III

Shared genes between differentially expressed genes at 24 and $48 \mathrm{~h}$. GFOLD differential expression values are displayed from this study, and compared with differential expression values. GFOLD value is the normalized GFOLD $\log _{2}$ fold change value, the first RPKM (reads per kilobase of transcript per million mapped reads values) represents the susceptible mosquitoes, while the second RPKM corresponds to the refractory mosquitoes. Panel A is a comparison after $24 \mathrm{~h}$, while panel B is after $48 \mathrm{~h}$

A

\begin{tabular}{|c|c|c|c|c|c|}
\hline Vector base gene ID & $\begin{array}{l}\text { GFOLD } \\
\text { value }\end{array}$ & $\begin{array}{l}\log 2 \text { fold } \\
\text { change }\end{array}$ & FirstRPKM & SecondRPKM & Gene description \\
\hline AAEL015458*** & 1.63429 & 2.09472 & 0.482571 & 4.29899 & transferrin \\
\hline AAEL008019** & 1.15154 & 1.35835 & 8.45216 & 44.6763 & hypothetical protein \\
\hline AAEL006911* & 1.13906 & 1.33454 & 2.89728 & 15.0603 & microtubule-associated protein \\
\hline AAEL005091** & 0.962343 & 1.23939 & 2.49015 & 12.1359 & conserved hypothetical protein \\
\hline AAEL005561*** & 0.92409 & 1.06373 & 4.33529 & 18.661 & plasma membrane calcium-transporting ATPase 3 (pmca3) \\
\hline AAEL009762**** & 0.887696 & 1.40863 & 0.602798 & 3.33524 & cytochrome $\mathrm{P} 450$ \\
\hline AAEL006138**^ & 0.83831 & 0.886424 & 19.4911 & 74.1502 & hypothetical protein \\
\hline AAEL010434**^ & 0.800671 & 0.867546 & 10.7857 & 40.5015 & Vitellogenin-A1 Precursor (VG)(PVG1) \\
\hline AAEL000940*** & 0.71424 & 0.768284 & 130.763 & 458.353 & conserved hypothetical protein \\
\hline AAEL008413** & 0.709035 & 0.988036 & 1.21075 & 4.95468 & serine/threonine protein kinase \\
\hline AAEL001503* & 0.659975 & 0.751801 & 8.16197 & 28.2886 & sodium/hydrogen exchanger 3 (nhe3) \\
\hline AAEL003609** & 0.637787 & 0.917252 & 1.18354 & 4.61077 & neurobeachin \\
\hline AAEL003733** & 0.588112 & 0.805074 & 0.806103 & 2.90214 & hypothetical protein \\
\hline AAEL007817** & 0.571004 & 0.857399 & 0.659328 & 2.46414 & hypothetical protein \\
\hline AAEL008234***^ & 0.565555 & 1.03674 & 0.559988 & 2.38295 & dishevelled \\
\hline AAEL017241** & 0.540569 & 0.922035 & 1.62698 & 6.37326 & \\
\hline AAEL006126**^ & 0.528522 & 0.597776 & 7.44993 & 23.2034 & conserved hypothetical protein \\
\hline AAEL006563**^ & 0.49487 & 0.670025 & 7.09483 & 23.2468 & Vitellogenic carboxypeptidase Precursor (EC 3.4.16.-) \\
\hline AAEL008216*** & 0.468322 & 0.548729 & 15.6068 & 46.9852 & aconitase \\
\hline AAEL003331*** & 0.389598 & 1.01092 & 0.214398 & 0.901404 & hypothetical protein \\
\hline AAEL008853*** & 0.363469 & 0.515272 & 7.3669 & 21.6768 & choline/ethanolamine kinase \\
\hline AAEL000191*** & 0.358468 & 0.492464 & 6.24993 & 18.0997 & conserved hypothetical protein \\
\hline AAEL006728***^ & 0.287245 & 0.577257 & 2.62092 & 8.06135 & ubiquitin-conjugating enzyme E2 c \\
\hline AAEL013074***^ & 0.281054 & 0.373851 & 20.7738 & 55.4011 & adenylyl cyclase-associated protein \\
\hline AAEL006785*** & 0.275308 & 0.314515 & 210.798 & 539.46 & 60 S ribosomal protein L18a \\
\hline AAEL009630** & 0.267701 & 0.519107 & 1.76691 & 5.21699 & high-affinity cgmp-specific 3,5-cyclic phosphodiesterase \\
\hline AAEL005358**** & 0.266146 & 0.571726 & 0.924488 & 2.83316 & conserved hypothetical protein \\
\hline AAEL000087*** & 0.230243 & 1.8899 & 0.020329 & 0.178293 & macroglobulin/complement \\
\hline AAEL001972*** & 0.213241 & 0.571861 & 3.44608 & 10.5697 & TATA box binding protein (TBP)-associated factor, putative \\
\hline AAEL004699*** & 0.207845 & 0.258941 & 34.393 & 84.6913 & conserved hypothetical protein \\
\hline AAEL008329*** & 0.187807 & 0.239528 & 207.059 & 503.059 & 60 S ribosomal protein L24 \\
\hline AAEL011326***^ & 0.170562 & 0.563302 & 0.751053 & 2.29116 & conserved hypothetical protein \\
\hline AAEL011756*** & 0.157017 & 0.213465 & 68.2496 & 162.847 & aldehyde dehydrogenase \\
\hline AAEL013614***^ & 0.138192 & 0.220292 & 8.36298 & 20.0499 & clathrin heavy chain \\
\hline AAEL005706*** & 0.133593 & 0.491582 & 0.733715 & 2.12793 & triacylglycerol lipase \\
\hline AAEL013694*** & 0.112597 & 0.149466 & 277.193 & 632.684 & 40S ribosomal protein SA \\
\hline AAEL001898*** & 0.08628 & 0.243694 & 3.39235 & 8.26768 & conserved hypothetical protein \\
\hline AAEL006511***^ & 0.076473 & 0.117664 & 374.017 & 835.071 & ubiquitin \\
\hline AAEL001158*** & 0.035571 & 0.379164 & 1.78576 & 4.78794 & fructose-1,6-bisphosphatase \\
\hline AAEL001516***^ & 0.008267 & 0.172471 & 3.04556 & 7.06473 & vesicle associated protein, putative \\
\hline AAEL011900** & 0.007043 & 0.398599 & 1.41796 & 3.85581 & $\mathrm{~N}$-acetyllactosaminide beta-1,3-Nacetylglucosaminyltransferase, putative \\
\hline AAEL000026***^ & -0.02263 & -0.02764 & 0.382792 & 0.775454 & dynein light chain, putative \\
\hline AAEL002813*** & -0.02916 & -0.15403 & 56.2173 & 103.968 & coupling factor, putative \\
\hline AAEL013252*** & -0.08415 & -0.34997 & 2.47179 & 3.98877 & hypothetical protein \\
\hline AAEL013407***^ & -0.08858 & -0.13579 & 64.4558 & 120.724 & catalase \\
\hline AAEL007293***^ & -0.115 & -0.28768 & 6.79592 & 11.4547 & cAMP-dependent protein kinase catalytic subunit \\
\hline AAEL011476***^ & -0.13368 & -0.6027 & 1.91153 & 2.58157 & conserved hypothetical protein \\
\hline AAEL009275*** & -0.13769 & -0.29124 & 12.5854 & 21.1615 & protein phosphatase-1 \\
\hline AAEL009658*** & -0.15448 & -0.29219 & 6.7785 & 11.3903 & alpha,alpha-trehalase \\
\hline
\end{tabular}




\begin{tabular}{|c|c|c|c|c|c|}
\hline AAEL013979*** & -0.21581 & -0.6126 & 1.88957 & 2.5368 & conserved hypothetical protein \\
\hline AAEL015312**** & -0.25461 & -0.38054 & 23.8955 & 37.7669 & cathepsin b \\
\hline AAEL004181** & -0.26924 & -0.45802 & 1.12191 & 1.68004 & conserved hypothetical protein \\
\hline AAEL002793*** & -0.32904 & -0.66303 & 1.2945 & 1.67913 & conserved hypothetical protein \\
\hline AAEL001432*** & -0.34173 & -0.41512 & 39.1863 & 60.4723 & protein disulfide isomerase \\
\hline AAEL012245**** & -0.44171 & -2.28002 & 0.326285 & 0.109783 & conserved hypothetical protein \\
\hline AAEL003067**** & -0.45827 & -1.09195 & 1.07278 & 1.02269 & conserved hypothetical protein \\
\hline AAEL002759*** & -0.46062 & -0.5438 & 17.766 & 25.0759 & tropomyosin invertebrate \\
\hline AAEL004958**** & -0.46068 & -3.56953 & 0.110887 & 0 & conserved hypothetical protein \\
\hline AAEL012349**** & -0.46068 & -3.56953 & 0.154968 & 0 & lipase 1 precursor \\
\hline AAEL013566**** & -0.48876 & -1.91745 & 0.978856 & 0.494022 & C-Type lectin (CTL) - galactose binding \\
\hline AAEL015004*** & -0.51611 & -0.79771 & 3.27493 & 3.87027 & hypothetical protein \\
\hline AAEL004027*** & -0.59755 & -0.70559 & 26.8693 & 33.8975 & glucose dehydrogenase \\
\hline AAEL014190**** & -0.59899 & -2.52514 & 0.189295 & 0.048122 & elongase, putative \\
\hline AAEL009244*** & -0.61831 & -0.6692 & 195.675 & 253.206 & serine-type enodpeptidase \\
\hline AAEL013853**** & -0.92413 & -1.80399 & 0.947571 & 0.535306 & C-Type Lectin (CTL) - galactose binding \\
\hline AAEL013648**** & -0.93159 & -2.59552 & 0.293348 & 0.08286 & conserved hypothetical protein \\
\hline AAEL001295**** & -1.03599 & -1.58042 & 1.66246 & 1.1286 & conserved hypothetical protein \\
\hline AAEL002652*** & -1.14538 & -2.98457 & 0.129852 & 0.023579 & hypothetical protein \\
\hline AAEL007942***^ & -1.25268 & -1.58534 & 7.32334 & 4.99762 & fibrinogen and fibronectin \\
\hline AAEL017211** & -1.36322 & -1.89536 & 16.8411 & 9.17417 & cecropin anti-microbial peptide \\
\hline AAEL001287** & -1.5998 & -3.38312 & 0.620966 & 0.084568 & conserved hypothetical protein \\
\hline AAEL002796** & -1.78703 & -2.66264 & 0.730636 & 0.224168 & 1-asparaginase i \\
\hline AAEL008046** & -2.28638 & -2.73068 & 4.00053 & 1.22289 & rh antigen \\
\hline AAEL003290** & -3.47153 & -6.25985 & 1.0477 & 0 & cell wall protein DAN4 precursor, putative \\
\hline AAEL017110** & -5.13546 & -7.88489 & 7.924 & 0 & \\
\hline AAEL009888** & -5.69755 & -7.44101 & 1.44731 & 0.008596 & bumetanide-sensitive $\mathrm{Na}-\mathrm{K}-\mathrm{Cl}$ cotransport protein, putative \\
\hline
\end{tabular}

^: previously detected as differentially expressed (see below for more information), but our results show changes in the opposite direction; *: previously detected as differentially expressed in mosquitoes of the MOYO-S or MOYO-R strains infected with DENv2 Jam1409 $18 \mathrm{~h}$ post infection; ${ }^{(10)}$ **: previously detected as differentially expressed in Chetumal (CTM) mosquito midguts 1dpi with DENv2 Jam1409 or blood; ${ }^{(11)} * * *$ : previously detected as differentially expressed in mosquitoes of the MOYO-S or MOYO-D strains infected with DENv2 Jam1409 $24 \mathrm{~h}$ post infection; ${ }^{(14)} * * * *$ : previously detected as differentially expressed in mosquitoes of the Rockefeller strain infected with DENv2 New Guinea C $48 \mathrm{~h}$ post infection. ${ }^{(12)}$

B

\begin{tabular}{|c|c|c|c|c|c|}
\hline Vector base gene ID & $\begin{array}{l}\text { GFOLD } \\
\text { value }\end{array}$ & $\begin{array}{l}\log 2 \text { fold } \\
\text { change }\end{array}$ & FirstRPKM & SecondRPKM & Gene description \\
\hline AAEL008392***^ & 0.812048 & 1.3925 & 0.932716 & 2.58007 & conserved hypothetical protein \\
\hline AAEL006291*** & 0.57612 & 0.754547 & 4.46132 & 7.84022 & cullin \\
\hline AAEL010798***^ & 0.303677 & 0.440852 & 14.8129 & 20.9368 & ubiquitin-conjugating enzyme $\mathrm{E} 2 \mathrm{~g}$ \\
\hline AAEL000604***^ & 0.281466 & 0.478478 & 4.90627 & 7.11891 & hypothetical protein \\
\hline AAEL014190**** & -0.07837 & -2.18723 & 0.160454 & 0.020175 & elongase \\
\hline AAEL001295**** & -0.10263 & -0.4388 & 4.09937 & 3.14096 & conserved hypothetical protein \\
\hline AAEL004809**** & -0.12691 & -0.78669 & 2.839 & 1.68835 & conserved hypothetical protein \\
\hline AAEL002908**** & -0.27134 & -1.89772 & 1.03566 & 0.250423 & hypothetical protein \\
\hline AAEL002818*** & -0.31054 & -0.62888 & 3.96005 & 2.6588 & splicing factor u2af large subunit \\
\hline AAEL014035***^ & -0.43611 & -0.7278 & 2.89018 & 1.81228 & suppressor of actin (sac) \\
\hline AAEL002889**** & -0.51237 & -1.01956 & 4.71438 & 2.3963 & hypothetical protein \\
\hline AAEL007075***^ & -0.75562 & -1.15049 & 3.75319 & 1.74852 & conserved hypothetical protein \\
\hline AAEL001737**** & -0.97528 & -2.78669 & 0.35227 & 0.036911 & conserved hypothetical protein \\
\hline AAEL008546**** & -1.02804 & -3.97572 & 1.07999 & 0 & conserved hypothetical protein \\
\hline AAEL012326*** & -1.08918 & -1.18633 & 234.609 & 107.293 & calmodulin \\
\hline AAEL002696**** & -1.18611 & -1.73636 & 1.56737 & 0.480957 & hypothetical protein \\
\hline AAEL002023***^ & -1.53962 & -1.7826 & 12.42 & 3.74596 & imaginal disc growth factor \\
\hline AAEL011851***^ & -2.09883 & -3.61532 & 1.81474 & 0.125029 & conserved hypothetical protein \\
\hline
\end{tabular}

$\wedge$ : previously detected as differentially expressed (see below for more information), but our results show changes in the opposite direction; ***: previously detected as differentially expressed in mosquitoes of the MOYO-S or MOYO-D strains infected with DENv2 Jam1409 48 $\mathrm{h}$ post infection; (14) ****: previously detected as differentially expressed in mosquitoes of the Rockefeller strain infected with DENv2 New Guinea $\mathrm{C} 48 \mathrm{~h}$ post infection. ${ }^{(12)}$ 
Conversely, we observed a higher transcript expression of CTL, a c-type lysozyme (AAEL003712) in refractory mosquitoes. Lysozymes have historically been implicated as anti-bacterial agents. When lysozyme-c was silenced, mosquitoes had a higher titre of dengue virus, suggesting that lysozymes may exert an inhibitory effect on the virus itself. ${ }^{(23)}$

We found higher expression of autophagy related genes, which are normally associated with organelle recycling and destruction, but recently have been implicated in reducing viral titres. ${ }^{(29)}$ The opposite seems to be true for DENV infections, where autophagy related genes (APGs) augment viral infection and replication. ${ }^{(27)}$ Silencing Aedronc, an initiator caspase, decreased autophagy and DENV titres in Ae. aegypti, suggesting an apoptotic basis of autophagy control. (29) DENV may induce autophagy and subsequent autophagosome formation, using virus induced double membrane vesicles as replication sites, ${ }^{(27)}$ although mechanisms using lipid metabolism, lipid droplets, virion maturation and dsRNA localization also have been proposed.(15) This pro-viral effect is consistent with reports describing significant increases in APG expression in susceptible mosquitoes exposed to DENV. (7, $, 11,13)^{2}$ There is a trend in DENVrefractory mosquitoes to have increased expression of Inhibitor of Apoptosis (IAP), Buffy, and anti-apoptotic genes, ${ }^{(9,13)}$ suggesting that the autophagy pathway may contribute to the DENV refractory phenotype.

A limitation of this study is that the experiments were not fully replicated; material from multiple replicates were pooled for the RNA sequencing, and therefore we analyzed the data with multiple programs and approaches for replicated and non-replicates experiments. All approaches identified the same genes. We identified specific genes that were over- or under-expressed in Cali-MIB or Cali-S mosquitoes after exposure to dengue virus. The results on the differential expression of specific genes identified using the RNAseq approach were confirmed using cDNAs generated from the same RNAs used to make the libraries, but also were confirmed using cDNAs generated from independently selected MIB and $\mathrm{S}$ lines of mosquitoes.

It is evident that there are proximate differences in DENV processing by Cali-S and Cali-MIB females, although the rationale is unclear because most studies suggest very little or no significant impact of DENV on overall Ae. aegypti fitness. ${ }^{(30)}$ Whether these responses are restricted to DENV, to other flaviviruses such as Zika, and yellow fever or to other arboviruses such as Chikungunya, will help us understand the extent of differential gene expression as a general antiviral response in Ae. aegypti. Future studies will use RNAi based gene knockdown studies to examine the phenotypic function of candidate genes identified in this study. We also will use DNA-based genetic analyses to separate inherent genetic differences between the strains from their differential responses to DENV.

Data availability - All relevant data are within the paper and its Supplementary data. The raw sequencing data as well as processed differential expression data is available to the public through NCBI's Gene Expression Omnibus (GEO) database (GSE90974).

\section{ACKNOWLEDGEMENTS}

To the Vector Biology and Control team at CIDEIM for training and technical support, and Rachel Blair and Iman Baharmand (SFU) for technical assistance.

\section{AUTHORS' CONTRIBUTION}

$\mathrm{CL}$ and $\mathrm{CBO}$ designed the study; PAC and $\mathrm{CBO}$ selected the strains and infected the mosquitoes; PAC and CL extracted RNA and prepared libraries; CL and HC sequenced the libraries, did the DE analysis, and verification using ddPCR; HC, $\mathrm{PAC}, \mathrm{CBO}$ and $\mathrm{CL}$ wrote and edited the manuscript and prepared figures and tables.

\section{REFERENCES}

1. Mairuhu ATA, Wagenaar J, Brandjes DPM, Van Gorp ECM. Dengue: an arthropod-borne disease of global importance. Eur J Clin Microbiol. 2004; 23(6): 425-33.

2. Bhatt S, Gething PW, Brady O, Messina J, Farlow A, Moyes C, et al. The global distribution and burden of dengue. Nature. 2013; 496(7446): 504-7.

3. McGraw EA, O'Neill SL. Beyond insecticides: new thinking on an ancient problem. Nat Rev Microbiol. 2013; 11(3): 181-93.

4. Ocampo CB, Wesson DM. Population dynamics of Aedes aegypti from a dengue hyperendemic setting in Colombia. Am J Trop Med Hyg. 2004; 71(4): 506-13.

5. Serrato IM, Caicedo PA, Lowenberger CA, Ocampo C. Vector competence and innate immune responses to dengue virus infection in selected laboratory and field-collected Stegomyia aegypti (=Aedes aegypti). Med Vet Entomol. 2017; 31(3): 312-9.

6. Barón OL, Ursic-Bedoya R, Lowenberger CA, Ocampo CB. Differential gene expression from midguts of refractory and susceptible lines of the mosquito, Aedes aegypti, infected with Dengue-2 virus. J Insect Sci. 2010; 10: 41.

7. Ocampo CB, Caicedo PA, Jaramillo G, Ursic Bedoya R, Baron $\mathrm{O}$, Serrato IM, et al. Differential expression of apoptosis related genes in selected strains of Aedes aegypti with different susceptibilities to dengue virus. PLoS One. 2013; 8(4): e61187.

8. Caicedo P, Barón O, Pérez M, Alexander N, Lowenberger C, Ocampo C. Selection of Aedes aegypti (Diptera: Culicidae) strains that are susceptible or refractory to Dengue-2 virus. Can Entomol. 2013; 145(3): 273-82.

9. Sim S, Jupatanakul N, Ramirez JL, Kang S, Romero-Vivas CM, Mohammed H, et al. Transcriptomic profiling of diverse Aedes aegypti strains reveals increased basal-level immune activation in dengue virus-refractory populations and identifies novel virus-vector molecular interactions. PLoS Negl Trop Dis. 2013; 7(7): e2295.

10. Behura S, Gomez-Machorro C, Harker B, DeBruyn B, Lovin D, Hemme R, et al. Global cross-talk of genes of the mosquito Aedes aegypti in response to dengue virus infection. PLoS Negl Trop Dis. 2011; 5(11): e1385.

11. Bonizzoni M, Dunn WA, Campbell CL, Olson KE, Marinotti O, James A. Strain variation in the transcriptome of the dengue fever vector, Aedes aegypti. G3 (Bethesda). 2012; 2(1): 103-14.

12. Colpitts T, Cox J, Vanlandingham D, Feitosa F, Cheng G, Kurscheid S, et al. Alterations in the Aedes aegypti transcriptome during infection with West Nile, dengue and yellow fever viruses. PLoS Pathog. 2011; 7(9): e1002189.

13. Behura S, Gomez-Machorro C, DeBruyn B, Lovin D, Harker B, Romero-Severson $\mathrm{J}$, et al. Influence of mosquito genotype on transcriptional response to dengue virus infection. Funct Integr Genomic. 2014; 14(3): 581-9.

14. Chauhan C, Behura S, DeBruyn B, Lovin D, Harker B, Gomez- 
Machorro C, et al. Comparative expression profiles of midgut genes in dengue virus refractory and susceptible Aedes aegypti across critical period for virus infection. PLoS One. 2012; 7(10): e47350.

15. Palmer WH, Varghese FS, van Rij RP. Natural variation in resistance to virus infection in dipteran insects. Viruses. 2018; 10(3): 1-37.

16. Jupatanakul N, Sim S, Dimopoulos G. Aedes aegypti ML and Niemann-Pick type $\mathrm{C}$ family members are agonists of dengue virus infection. Dev Comp Immunol. 2014; 43(1): 1-9.

17. Smartt CT, Erickson JS. Expression of a novel member of the odorant-binding protein gene family in Culex nigripalpus (Diptera: Culicidae). J Med Entomol. 2009; 46(6): 1376-81.

18. Marquardt WC. Biology of disease vectors. 2nd ed. Amsterdam: Elsevier Inc.; 2005.

19. Bonizzoni M, Dunn W, Campbell C, Olson K, Dimon M, Marinotti $\mathrm{O}$, et al. RNA-seq analyses of blood-induced changes in gene expression in the mosquito vector species, Aedes aegypti. BMC Genomics. 2011; 12: 82.

20. Caicedo PA, Serrato IM, Sim S, Dimopoulos G, Coatsworth H, Lowenberger $\mathrm{C}$, et al. Immune response-related genes associated to blocking midgut dengue virus infection in Aedes aegypti strains that differ in susceptibility. Insect Sci. 2018; 26(4): 635-48.

21. Leaungwutiwong P, Kelley JF, Sachair A, Jittmittraphap A, Luplertlop N. Relationship between MMP expression and virulence of dengue virus type- 2 in infected mosquito and mammalian cells. Jpn J Infect Dis. 2016; 69(1): 45-50.

22. Kantor AM, Dong S, Held NL, Ishimwe E, Passarelli AL, Clem $\mathrm{RJ}$, et al. Identification and initial characterization of matrix metalloproteinases in the yellow fever mosquito, Aedes aegypti. Insect Mol Biol. 2017; 26(1): 113-26.

23. Ramirez JL, Souza-Neto J, Cosme RT, Rovira J, Ortiz A, Pascale $\mathrm{JM}$, et al. Reciprocal tripartite interactions between the Aedes aegypti midgut microbiota, innate immune system and dengue virus influences vector competence. PLoS Negl Trop Dis. 2012; 6(3): 1-11.

24. Brackney DE, Foy BD, Olson KE. The effects of midgut serine proteases on dengue virus type 2 infectivity of Aedes aegypti. Am J Trop Med Hyg. 2008; 79(2): 267-74.

25. Molina-Cruz A, Gupta L, Richardson J, Bennett K, Black IVW, Barillas-Mury C. Effect of mosquito midgut trypsin activity on dengue-2 virus infection and dissemination in Aedes aegypti. Am J Trop Med Hyg. 2005; 72(5): 631-7.

26. Guo X, Xu Y, Bian G, Pike AD, Xie Y, Xi Z. Response of the mosquito protein interaction network to dengue infection. BMC Genomics. 2010; 11(1): 1-15.
27. Lee HK, Iwasaki A. Autophagy and antiviral immunity. Curr Opin Immunol. 2008; 20(1): 23-9.

28. Gillen CM, Blair CR, Heilman NR, Somple M, Stulberg M, Thombre R, et al. The cation-chloride cotransporter, masBSC, is widely expressed in Manduca sexta tissues. J Insect Physiol. 2006; 52(7): 661-8.

29. Eng MW, van Zuylen MN, Severson DW. Apoptosis-related genes control autophagy and influence DENV-2 infection in the mosquito vector, Aedes aegypti. Insect Biochem Mol Biol. 2016; 76: 70-83.

30. Lambrechts L, Scott TW. Mode of transmission and the evolution of arbovirus virulence in mosquito vectors. Proc Biol Sci. 2009; 276(1660): 1369-78.

31. Shertz CA, Cardenas ME. Exploiting and subverting tor signaling in the pathogenesis of fungi, parasites, and viruses. PLoS Pathog. 2011; 7(9): 8-10.

32. Paluzzi JP, O’Donnell MJ. Identification, spatial expression analysis and functional characterization of a pyrokinin-1 receptor in the Chagas' disease vector, Rhodnius prolixus. Mol Cell Endocrinol. 2012; 363(1-2): 36-45.

33. Ternes P, Franke S, Zähringer U, Sperling P, Heinz E. Identification and characterization of a sphingolipid $\Delta 4$-desaturase family. J Biol Chem. 2002; 277(28): 25512-8.

34. Cheng J, Wang Y, Li F, Liu J, Sun Y, Wu J. Cloning and characterization of a mannose binding C-type lectin gene from salivary gland of Aedes albopictus. Parasit Vectors. 2014; 7(1): 337.

35. Hussain M, Asgari S. MicroRNA-like viral small RNA from dengue virus 2 autoregulates its replication in mosquito cells. Proc Natl Acad Sci USA. 2014; 111(7): 2746-51.

36. Heaton NS, Randall G. Dengue virus-induced autophagy regulates lipid metabolism. Cell Host Microbe. 2010; 8(5): 422-32.

37. Lee Y-R, Hu H-Y, Kuo S-H, Lei H-Y, Lin Y-S, Yeh T-M, et al. Dengue virus infection induces autophagy: an in vivo study. J Biomed Sci. 2013; 20(1): 65.

38. Clements AN. The biology of mosquitoes: transmission of viruses and interactions with bacteria. Vol. 3. Oxfordshire: CABI; 2012.

39. van Gorp EC, Suharti C, Mairuhu AT, Dolmans WM, van Der Ven J, Demacker PN, et al. Changes in the plasma lipid profile as a potential predictor of clinical outcome in dengue hemorrhagic fever. Clin Infect Dis. 2002; 34(8): 1150-3.

40. Gillen C, Akuma D, Piermarini P. Expression pattern and RNAi inhibition of putative sodium-coupled cation-chloride cotransporters in the mosquito Aedes aegypti. Faseb J. 2015; 29(Suppl. 1). 